\title{
Surface density of growth defects in different PVD hard coatings prepared by sputtering
}

\author{
P. Panjan ${ }^{\mathrm{a}, *}$, M. Čekada ${ }^{\mathrm{a}}$, M. Panjan ${ }^{\mathrm{a}}$, D. Kek-Merl ${ }^{\mathrm{a}}$, F. Zupanič ${ }^{\mathrm{b}}$, L. Čurkovićc $^{\mathrm{c}}$, S. Paskvale ${ }^{\mathrm{a}}$ \\ a Jožef Stefan Institute, Jamova 39, 1000 Ljubljana, Slovenia \\ ${ }^{\mathrm{b}}$ University of Maribor, Faculty of Mechanical Engineering, Smetanova 17, 2000 Maribor, Slovenia \\ ${ }^{\mathrm{c}}$ Faculty of Mechanical Engineering and Naval Architecture, Zagreb, Croatia
}

Keywords:

PVD coatings

Defects

\begin{abstract}
A B S T R A C T
Growth defects are present in all PVD hard coatings. They have detrimental influence on their tribological properties (higher sticking of workpiece material, higher friction coefficient, worse corrosion resistance, higher gas permeation). In order to improve the tribological properties of PVD hard coatings it is important to minimize the concentration of growth defects. Conventional TiAlN single layer as well as AlTiN/TiN and TiAIN/CrN nanolayer coatings were deposited on cemented carbide, powder metallurgical high speed steel (ASP30) and cold work tool steel (D2) by magnetron sputtering in the CC800/7 and CC800/9 sinOx ML (CemeCon) deposition systems, respectively. The surface morphology of the coated substrates was examined by scanning electron microscope (FE-SEM) in combination with focused ion beam (FIB), and 3D stylus profilometer. By means of 3D-profilometry we performed several measurements and detailed analysis on a series of samples from the several hundred production batches. The influence of growth defects on GDOES (glow-discharge optical emission spectrometry) depth resolution and pitting corrosion was also studied.
\end{abstract}

(c) 2011 Elsevier Ltd. All rights reserved.

\section{Introduction}

Coated surfaces contain sporadic defects such as peaks and craters. Growth defects are inherent also in PVD hard coatings. The defect density can be reduced by proper substrate pretreatment and selection of optimal deposition parameters, however, it is difficult to eliminate them completely. The most common type of growth defects is nodular defects. The origin of such defects is irregularities on the substrate surface larger than $0.1 \mu \mathrm{m}$ (asperities, microscratces, inclusions, impurities) and especially the submicrometer sized particles which remain on the surface after the cleaning procedure or those which are generated during coating process. Such small particles nucleate the defects when the surface is coated.

The formation of nodular defects in PVD coatings is caused by shadowing effects initiated by seed particles or asperities on substrate surface during the deposition process because evaporation and sputtering are line-of-sight processes [1]. At the defect nucleation sites an accelerated growth relative to the matrix growth occurs. The diameter of the nodule, which is conical or parabolic in shape (depending on the flux distribution of the incoming species), increases with the coating thickness.

\footnotetext{
* Corresponding author.

E-mail address: peter.panjan@ijs.si (P. Panjan).
}

In order to obtain a smooth coating we have to eliminate particles in the production environment. To approach this goal various measures can be taken [2]. The most important one is a high-quality substrate cleaning procedure. The deposition system is also a strong generator of small particles which could contaminate the growing coating. Namely, coating is deposited also on the substrate holders, shields and on other parts at the inner wall of the vacuum chamber and after some deposition time this coating can be delaminated from the surface by stress release (thermal stress, internal compressive stress). The chips are transferred to the substrate surface and incorporated in the growing film. They are held to the substrate by electrostatic forces, which are much stronger than the gravitational forces on micrometer sized particles.

From this point of view a periodical cleaning of all components in the vacuum chamber is very important. The thickness of the deposit on inner parts of the vacuum chamber must be minimized in order to avoid the fracture of the stressed coatings. It is also important to avoid any turbulence in the gas flow, thus slow pumpdown and venting must be used. Additional problems are the wear particles from moving surfaces in contact. The concentration of such particles can be reduced if the moving components of the vacuum chamber are made of nongalling materials. Proper design of the fixturing holders is also very important - face upward mounting of substrates should be avoided. The angular distribution 
of the impinging vapor flux on the substrate surface has also a big influence on the growth defects. The more random the flux direction is the smaller the number and size of pinholes.

One of possible origins of dust particles is their formation in plasma. Researchers in the semiconductor industry found that the contamination of silicon wafers occurred not only due to poor cleaning procedure and during handling of wafers in the air, but it might be happening also inside the plasma processing reactors during etching or deposition [3-5]. Such submicrometer particles can be formed through the gas-phase reaction and aggregation of atoms or molecules from sputtering or etching processes. These particles are usually negatively charged in a typical glow discharge so they can levitate in RF plasma. During the process step when the plasma is switched off, they contaminate the substrate.

A large concentration of growth defects has a degrading effect on the mechanical properties, on the wear and corrosion resistance. Growth defects act like stress raisers, they increase the friction coefficient, increase the permeability for gases and decrease the wear and corrosion resistance of the coating [6,7].

In our previous papers [8-10] we described the types and origins of growth defects in PVD hard coatings. We used 3D stylus profilometry and FIB in combination with SEM for study of such growth defects.

In this paper the results of defect density measurements on nanolayered TiAlN/CrN and AlTiN/TiN coatings prepared by magnetron sputter deposition are presented. The results obtained on single layer TiAlN coating, prepared by magnetron sputter deposition are added for comparison. A large number of measurements have been performed in order to obtain adequate statistics.

The influence of growth defects on GDOES depth resolution and pitting corrosion was also studied.

\section{Experimental}

The magnetron sputtering system CC800/7 (CemeCon) was used for deposition of TiAlN coating, while TiN/TiAlN and TiAlN/CrN nanolayer hard coatings were deposited in CC800/9 sinOx ML (CemeCon). Both deposition systems are equipped with four unbalanced magnetron sources which are located pairwise in two sides of the chamber. The substrate holders enable 1-, 2- and 3-fold rotations. A powder metallurgical high speed steel (ASP30), a cold work tool steel (D2) and cemented carbide substrates were used as substrates. The substrates were polished, ultrasonically cleaned and dried in hot air. Prior to deposition they were cleaned by ion etching.

The surface morphology of the coatings was examined by 3D stylus profilometer (Taylor Hobson Talysurf). Its vertical resolution is a few tens of $\mathrm{nm}$, while the lateral resolution is limited to $1 \mu \mathrm{m}$. Any protruding peaks, even though their size is in the $100-\mathrm{nm}$ range, can easily be detected by the stylus. In contrast, the sensitivity for craters is lower. The measured area was $1 \mathrm{~mm} \times 1 \mathrm{~mm}$. For the applied precision ( $1 \mu \mathrm{m}$ in $x$-axis, $2 \mu \mathrm{m}$ in $y$-axis) the measurement time was about $2 \mathrm{~h}$. Thus we obtained a 3D image of the coating surface on a large scanning area with all the micrometer-sized details. The number of peaks (and holes) was evaluated based on different thresholds. We found that the optimal value is $0.5 \mu \mathrm{m}$ both for peaks and cavities.

A focused ion beam (FIB) workstation was used to prepare crosssection through the defects. We used FIB integrated in the FEI QUANTA 200 3D microscope. An ion beam was used to remove precise sections of the material (close to the defect) from the specimen surface by sputtering. The initial trough (with dimensions of approximately $8 \times 5 \times 4 \mu \mathrm{m}$ ) was milled at a high beam current $(20 \mathrm{nA})$, while the energy of ions was $30 \mathrm{keV}$. Then the cross-section was polished with a lower beam current ( $3 \mathrm{nA})$.
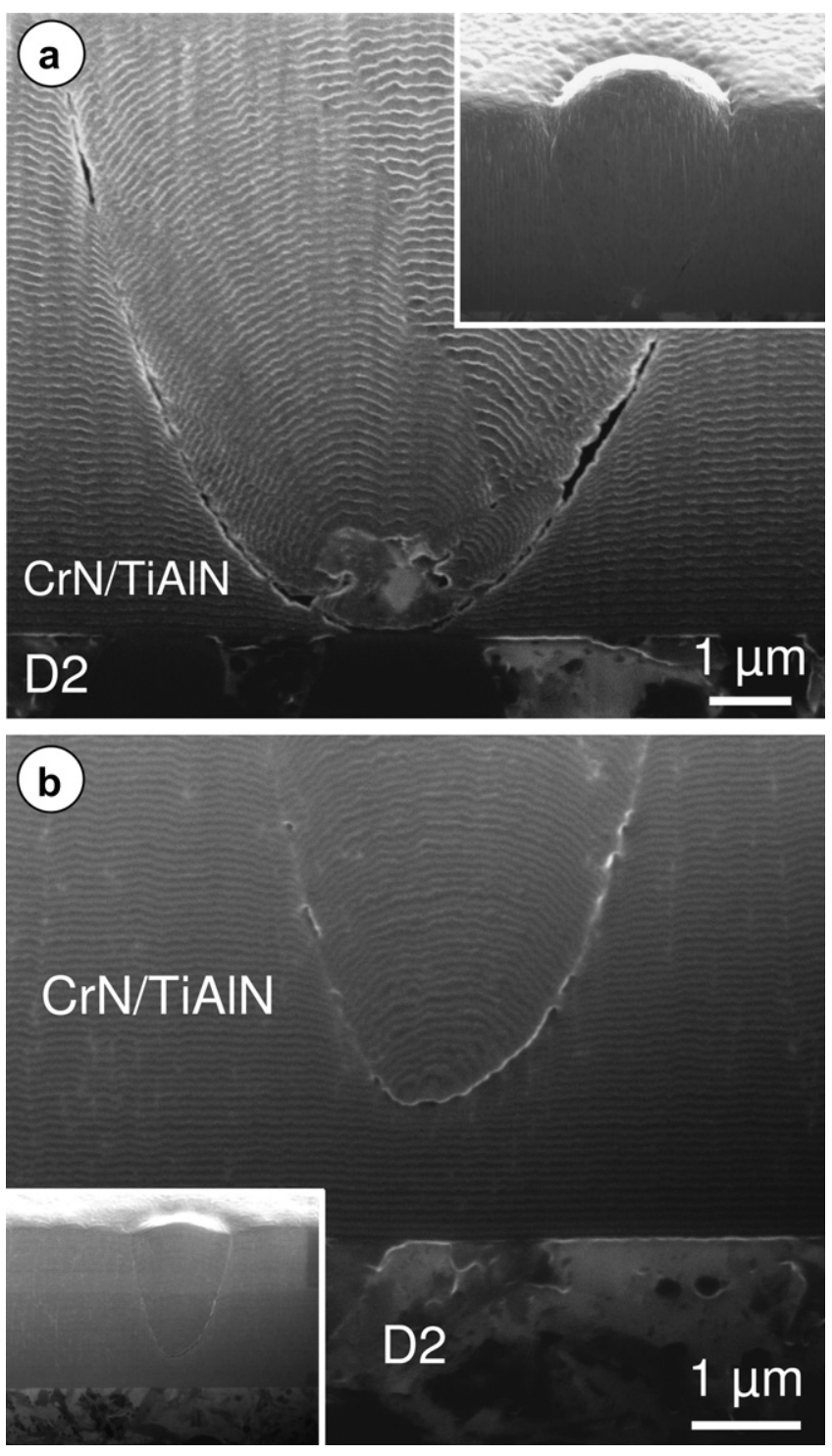

Fig. 1. SEM images of FIB cross-section of nodular defects in the TiAIN/CrN nanolayer coatings.

JEOL JSM-7600F field-emission microscope was used for the study of the coating microstructure and defect morphology in planar surface view and cross-sectional fracture view.

Compositional depth profiles were obtained by glow-discharge optical emission spectrometry (GDOES). We used Leco GDS-750 spectrometer with the anode of $4 \mathrm{~mm}$ diameter and sampling area of $12.5 \mathrm{~mm}^{2}$.

\section{Results}

Figs. 1 and 2 show FIB and SEM images of typical growth defects found in the TiAlN, TiAlN/CrN and AlTiN/TiN nanolayered coatings. Nanolayered coatings are especially useful for investigating the defects because the layer structure reveals the origin of the defects and their influence on the coating growth. SEM images on FIB cross section (Fig. 1) show that nodules have the form of a cone protruding above the coating surface. Nodular defect shown on Fig. 1a started to grow on the substrate surface on a seed which remained on the substrate surface. Fig. $1 \mathrm{~b}$ shows an example of a nodular defect which started to grow in the middle of the coating due to the incorporation of a foreign particle during the deposition process. 

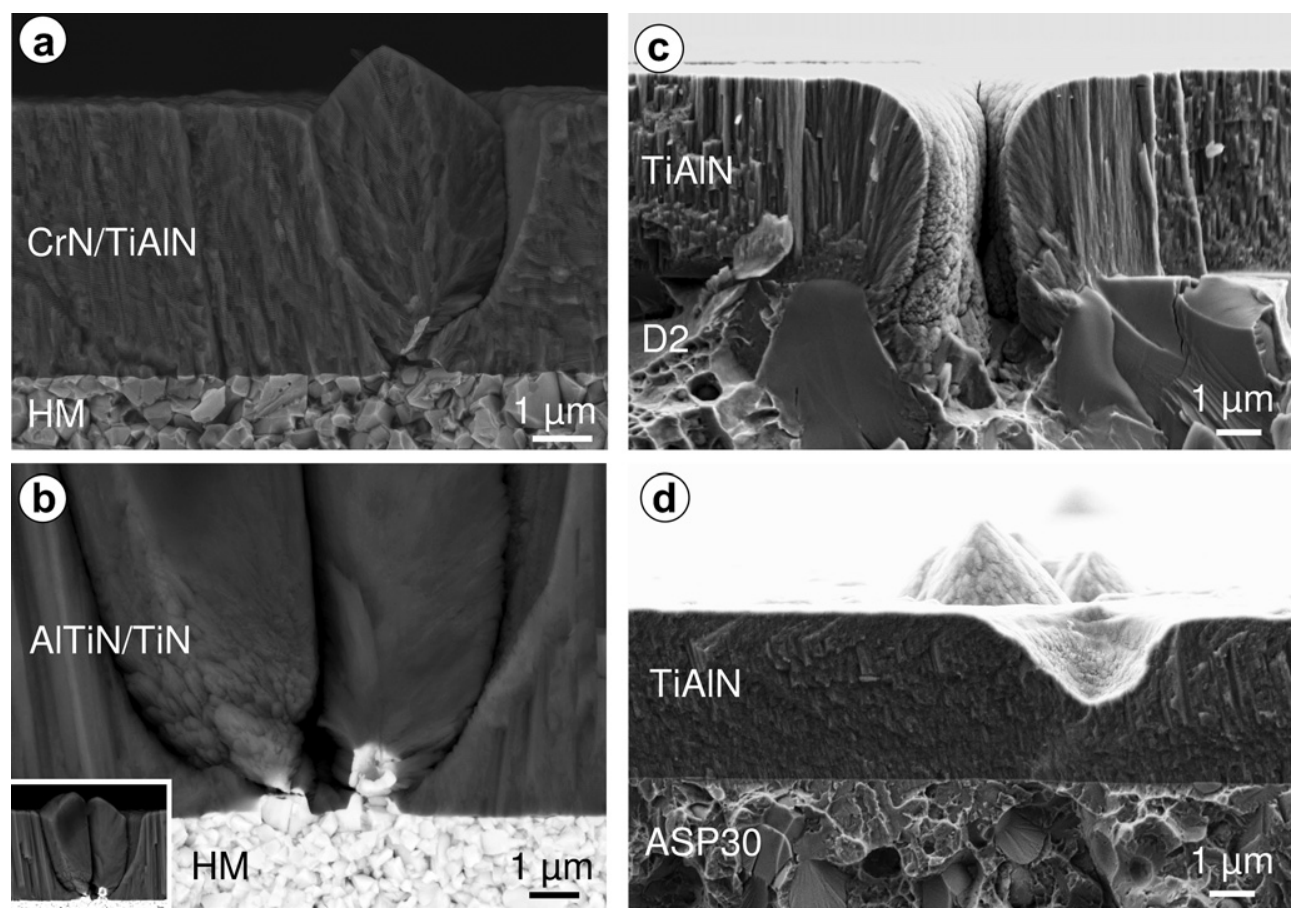

Fig. 2. SEM images of a nodular and pinhole defects in the TiAlN, TiAlN/CrN and AlTiN/TiN nanolayer coatings.

Fig. 2a shows cross-sectional backscattering electron micrographs of the TiAlN/CrN nanolayer deposited on cemented carbide. The seed which juts out from the substrate surface is clearly visible (in this case the seed is small part of tungsten carbide which
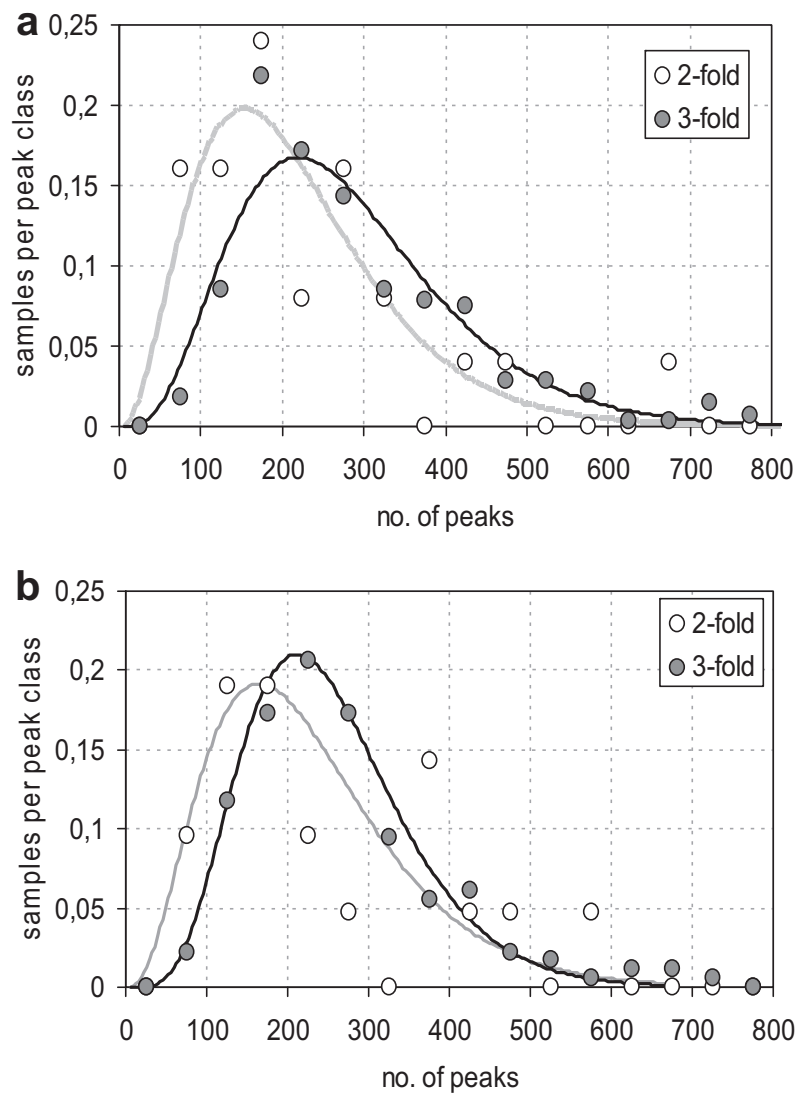

Fig. 3. Distribution o defect density for TiAlN (a) and AlTiN/TiN (b) nanolayer hard coatings deposited on cemented carbide substrates. remained on the substrate surface after mechanical pretreatment). An example of a very interesting nodular defect is shown on Fig. 2b. Cross-sectional SEM micrograph of the TiAIN/TiN nanolayer coating on cemented carbide shows that the nodular defect nucleated on two seeds (very small surface asperities, which remain on the substrate surface after its mechanical pretreatment). A typical internal structure of the nodules is also visible. The microstructure of the coating in the nodule is substantially different in comparison with that of the surrounding matrix. It can also be seen that the boundary between the cone-shaped defect and the coating is highly porous. Fig. 2c shows defects in the form of pinholes generated at the substrate pit (cavity), formed during polishing. It is too large to be closed by the deposited hard coating. On Fig. 2d there is an example of a dish-like hole forming when a coated foreign particle flakes off due to high stress in the coating.

We performed a series of measurements and detailed analysis on a series of samples from several hundred production batches. In each batch several substrates were put on different positions. A total of 500 samples coated with TiAlN and 230 samples coated with AlTiN/TiN nanolayer have been scanned using the 3D profilometer.

By scanning a standardized area and performing elementary statistical analysis, one can easily evaluate the density of defects for a desired coating. Each sample was measured twice, while some of them ten times. The scanning area was different for each measurement. The reproducibility for the same sample is quite

Table 1

Defect density (in $\mathrm{mm}^{-2}$ ) on the samples mounted at the same position on the holder which assured 1-, 2- and 3-fold rotations.

\begin{tabular}{|c|c|c|c|c|c|c|}
\hline \multirow{2}{*}{$\frac{\text { Coating }}{\text { Rotation type }}$} & \multicolumn{3}{|l|}{ TiAlN } & \multicolumn{3}{|c|}{ AlTiN/TiN } \\
\hline & 1 -fold & 2 -fold & 3 -fold & 1-fold & 2 -fold & 3-fold \\
\hline Batch 1 & 833 & 327 & 251 & 226 & 260 & 121 \\
\hline Batch 2 & 100 & 121 & 294 & 423 & 186 & 170 \\
\hline Batch 3 & 216 & 158 & 195 & 195 & 229 & 275 \\
\hline Batch 4 & 174 & 157 & 236 & 342 & 364 & 222 \\
\hline Batch 5 & 1110 & 387 & 428 & 325 & 420 & 210 \\
\hline
\end{tabular}




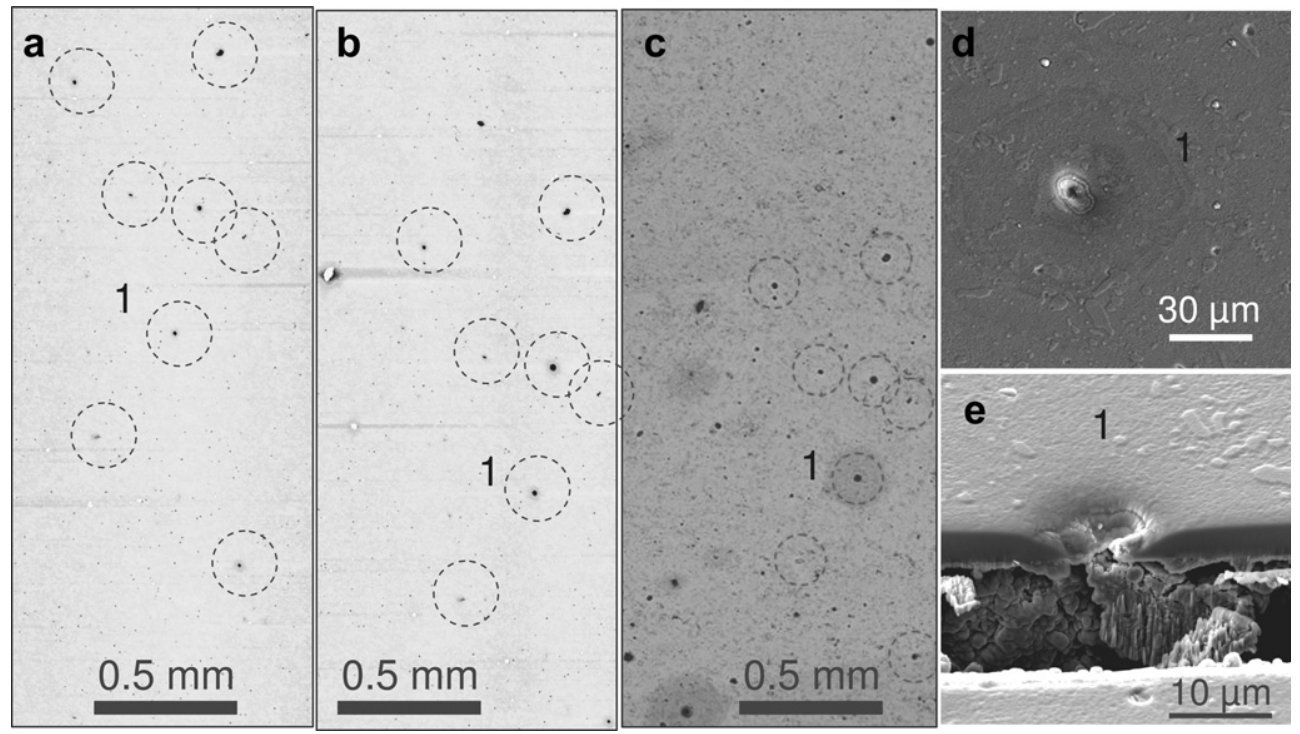

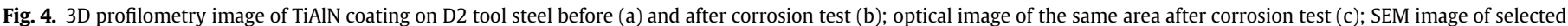
pinhole (number 1); FIB image of the pin-hole (number 1) cross-section after corrosion by backscattered electrons (e).

good. However, a strong scattering of results was observed on different samples from the same batch and especially on samples from different batches. On small scale (below $100 \mu \mathrm{m} \times 100 \mu \mathrm{m}$ ) the growth defects of various sizes are typically non-uniformly distributed over the coating surface and their surface density is typically several tens up to a thousand defects per $\mathrm{mm}^{2}$. On large scale $(1 \mathrm{~mm} \times 1 \mathrm{~mm})$ the distribution of defects is rather uniform. The number of peaks exceeds the number of holes by an order of magnitude. The deepest holes are half-size in comparison to the highest peaks.

From a large number of measurements we performed an elementary statistical analysis in order to evaluate the defect surface density for a desired coating (Fig. 3). The number of measurements is quite large (500 in total) and the number of defects per area often scatters considerably in the same series of samples. Therefore a statistical analysis is necessary to extract the overall results. A suitable distribution has to be fitted to the acquired measurements rather than taking a simple average where isolated outliers can spoil the final value considerably.

First we reduced the number of measurements to one measurement per sample by calculating the average. This was necessary to exclude biasing towards samples where more measurements have been done. Then we divided the samples in classes with a width of 50 defects $/ \mathrm{mm}^{2}$ ranging from 0 to 800 defects $/ \mathrm{mm}^{2}$ (the number of samples with over 800 defects is negligible). For any series of samples we thus constructed 20 classes $(x, y)$ where $x$ is the median number of defects of the class and $y$ the number of samples falling into this class. A natural choice for analysis is the Poisson distribution. For fitting we used the following form:

$y=y_{0}\left(\frac{x}{x_{0}}\right)^{n} e^{-\frac{x}{x_{0}}}$

where $n, x_{0}$ and $y_{0}$ were varied to get the best fit. The peak of the distribution is located at $n \cdot x_{0}$.

Fig. 3a shows the distribution of all the samples coated with TiAlN, divided in two series based on the type of rotation. The 3 -fold rotation gives a good fit, which is partly a consequence of a large number of samples. The peak of the distribution is located at 220 defects $/ \mathrm{mm}^{2}$. In 2 -fold rotation there is more scattering but the overall form is still in good agreement with the Poisson distribution. The peak is located at a significantly lower value, at 150 peaks $/ \mathrm{mm}^{2}$. The same analysis was performed also on AlTiN/ TiN nanolayer coatings for samples prepared using 2- and 3-fold rotation. In comparison with TiAlN we did not find any significant difference in peak location. We also analyzed the samples mounted at the same position in the vacuum chamber on the holder which assured 1-, 2- and 3-fold rotations. We found (see Table 1) that the difference in defect density between all the three samples from the same batch was rather high (up to 200\%). Samples from different batches were analyzed and the results are randomly distributed regarding the rotation type. There can also be a substantial difference (up to 50\%) on the two faces of the same 3-fold rotated sample. The results of all these measurements show that the formation of significant share of the defects is spatially localized and sporadic.

Due to the weak boundary between the nodular defects and the surrounding material some of them can become separated from the coating leaving a cavity. The ejection of a nodulus appeared when the coating is thick enough or if it is exposed to mechanical forces (e.g. sliding). A high concentration of such pinholes increases the coating porosity. The pinholes and the open structure between the nodulus and the surrounding coating are responsible for pitting corrosion (Fig. 4). A corrosion tests of PVD coatings, using

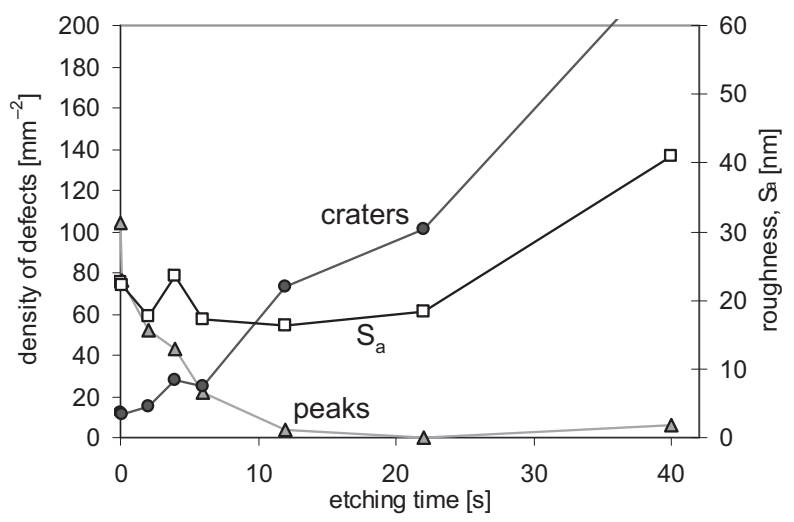

Fig. 5. Surface roughness, peak and crater density as a function of etching time during GDOES depth profiling of TiAIN hard coatings. 

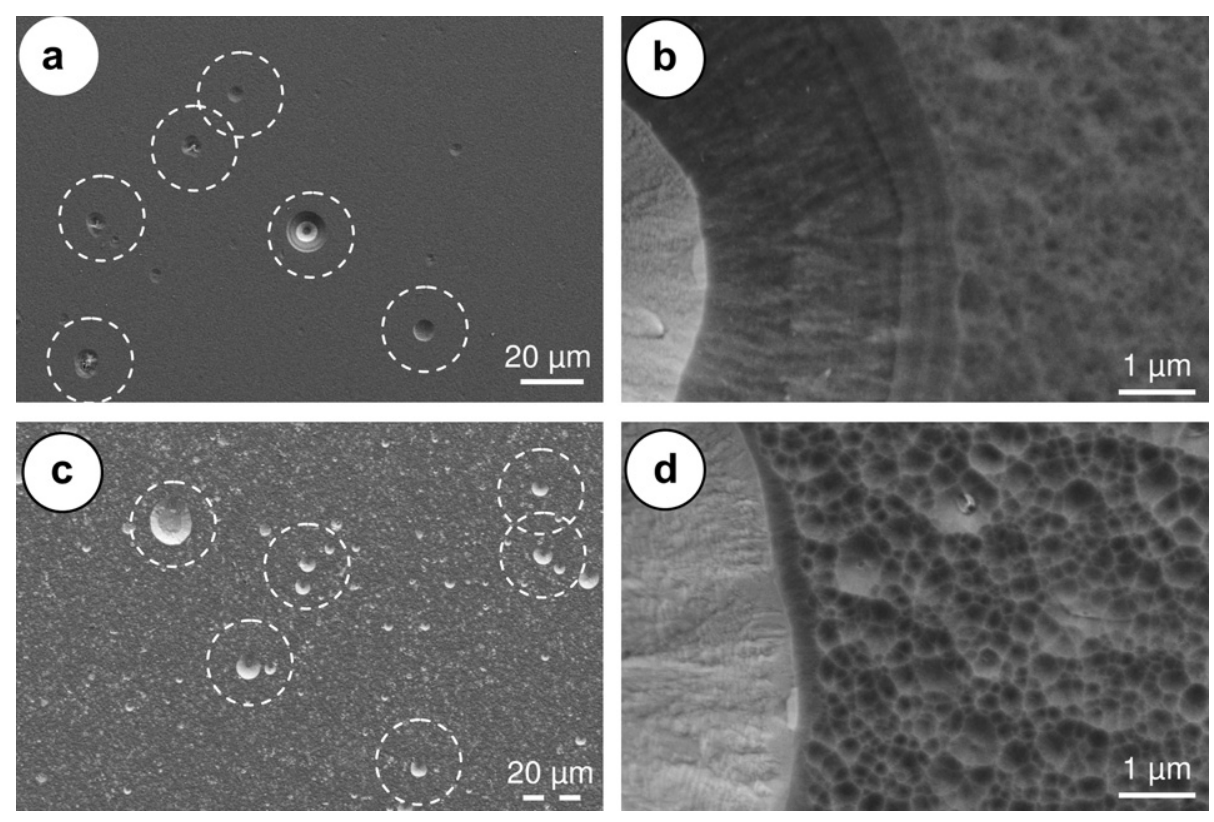

Fig. 6. SEM images of TiAIN hard coating in the GDOES crater after etching time of $6 \mathrm{~s}(\mathrm{a}, \mathrm{b})$ and $45 \mathrm{~s}$ (c, d).

electrochemical methods (potentiodynamic data and with electrochemical impedance spectroscopy), were performed in chloride medium. Surface characterization was performed before and after corrosion test by 3D-profilometry, optical microscopy and FIB. After corrosion we performed profilometry over the same area as on the as-deposited sample, paying special attention to the position of the pinholes at which pitting corrosion occurred (e.g. pinhole 1 on Fig. 4). Then we also took optical images of the same area (Fig. 4c). By comparison of the 3D profiles and the optical image it is possible to identify the network of pinholes also on the optical image. We selected individual pits on the coating surface and made a slice-byslice sectioning by FIB. The FIB image of pit (number 1) crosssections after corrosion is presented on Fig. 4e.

GDOES spectrometry is very often used for compositional depth profiling of hard coatings. The advantage of this technique is that it is very fast, but depth resolution is rather low. One reason for the loss of resolution with depth is due to nonuniform crater geometry. In practice, the crater bottom is curved during sputtering. Additional influences on depth resolution are from the growth defects built into the coating. We found that during ion etching various surface structures developed at such defects while they cause the perturbations of the sputtering process.

We performed compositional depth profiling of coatings using GDOES technique. The craters with different depths were prepared on selected coatings. The etching time was between 1 and $80 \mathrm{~s}$. After GDOES experiment we evaluated the coating topography of the GDOES crater bottom using the 3D profilometer. We found that after short ion etching (a few seconds) all cones formed during coating deposition process transformed into holes. Fig. 5 shows the density of peaks and holes as well as surface roughness of the TiAlN coating deposited on tool steel as a function of etching time. Density of peaks rapidly decreases after a short etching time, while the density of holes increases simultaneously. This effect can be explained by the reduction of the cohesive force between the inclusion and the coating after removal of the top layer and by high thermal stresses which appear during ion etching between the cones and the bulk coating. Namely, the electric field density on the conical defects is much higher compared to that of a flat surface. Thus an ion current with higher density appears which is responsible for higher local temperature and thermal stress. Such stresses can cause the detachment of the coated particles (see craters on Fig. 6a,c). We found that the surface roughness is more or less constant during etching of fine-grained coating with homogeneous composition while it rapidly increases when the steel substrate starts to etch. The reason for roughness increase is the generation of surface features during ion bombardment of inhomogeneous tool steel material.

\section{Conclusions}

From a large number of measurements with 3D stylus profilometer, we performed an elementary statistical analysis in order to evaluate the defect surface density for a desired coating. We found that the average concentration of defects on various substrates and for different rotation modes is comparable, while the scattering of the results is very high not only for the samples of different batches but also for the samples of the same batch. This means that we have different sources of dust particles inside of the vacuum chamber during the deposition process. We have to consider the influence of the fixturing holder components as well possible formation of dust particles in plasma. We also found that the growth defects are responsible for pitting corrosion as well as for low depth resolution of GDOES depth profiling.

\section{Acknowledgments}

This work was supported by the Slovenian Research Agency (projects L2-9189, L2-2100).

\section{References}

[1] Patten JW. Thin Solid Films 1979;63:121.

[2] Vetter J, Stuber M, Ulrich S. Surf Coat Technol 2003;168:169-78.

[3] Selwyn GS, Heidenreich JE, Haller KL. Appl Phys Lett 1990;57:1876-8.

[4] Merlino RL, Goree JA. Phys Today; July 2004:32-8.

[5] Lin I, Lai YJ, Juan WT, Chang MH, Chue HY. Vacuum 2002;66:285-91.

[6] Matox DM. SVC Bulletin; 2009:30-1. Spring.

[7] Ahn SH, Lee JH, Kim JG, Han JG. Surf Coat Technol 2004;177-178:638-44.

[8] Cekada M, Panjan P, Kek-Merl D, Panjan M, Kapun G. Vacuum 2008;82:252-6.

[9] Panjan P, Kek-Merl D, Zupanič F, Čekada M, Panjan M. Surf Coat Tech 2008, 202:2302-5.

[10] Panjan P, Čekada M, Panjan M, Kek-Merl D. Vacuum 2010;84:209-14. 